\title{
Role for adiponectin in effects of exercise in depression
}

New research in mice suggests that raised levels of adiponectin as a result of exercise modulate hippocampal neurogenesis, thus decreasing depression-like behaviours.

Previous work in rats has shown that voluntary running increases plasma levels of adiponectin, with a concurrent decrease in depression-like behaviours. However, the exact mechanisms underlying the positive effects of exercise on depression are unclear. These observations led a team of researchers from China to "explore whether adiponectin could be the key mediator of the antidepressant effect of physical exercise," explain corresponding authors Aimin Xu and Kwok-Fai So.

The researchers injected wildtype mice with adenoviruses expressing either adiponectin (Ad-Adn) or luciferase (Ad-Luc, the control). The mice underwent a range of behavioural tests (such as a forced swim test and a tail suspension test) to determine the level of depression-like behaviours. When compared with the Ad-Luc mice, the Ad-Adn mice showed notable decreases in depression-like behaviours in all the tests 2 weeks after receiving the injection. The Ad-Adn mice also showed considerable increases in hippocampal cell proliferation, the degree of which seemed to be associated with the extent of the reduction in depression-like behaviours.

$\mathrm{Xu}$, So and colleagues also demonstrated that adiponectin can cross the blood-brain

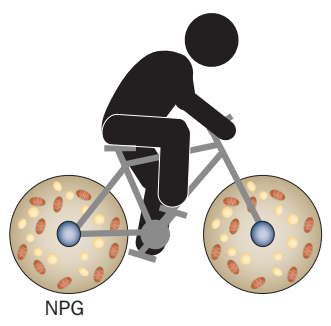

barrier. Adiponectin was injected into the tail vein of mice with a genetic knockout for adiponectin $\left(\right.$ Adipoq $\left.^{-/-}\right)$; $3 \mathrm{~h}$ later, adiponectin was detectable in the cerebrospinal fluid.

Running was found to increase hippocampal neurogenesis in wild-type mice. Whereas basal cell proliferation was unaffected in the Adipoq ${ }^{-/-}$mice, they showed no increase in hippocampal neurogenesis in response to running. These results suggest that adiponectin is required for the generation of new neurons in response to running and, therefore, seems to be a key factor in the positive effects of exercise on depression.

"Our findings raise a possibility of treating depression through increasing

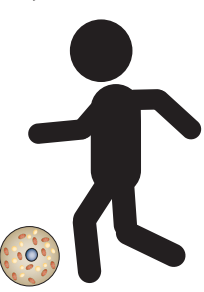
hippocampal levels of adiponectin using physical exercise or pharmaceutical agents," say Xu and So. They also suggest that their findings could explain the aetiology of other neurophysical diseases characterized by suppressed hippocampal neurogenesis and reduced levels of adiponectin, such as cognitive decline in patients with diabetes mellitus.

The team currently have several projects underway to build on these findings. They are repeating the experiments using other types of stress and hope to investigate whether the findings can be translated to humans.

Claire Greenhill

Original article Yau, S. Y. et al. Physical exercise-induced hippocampal neurogenesis and antidepressant effects are mediated by the adipocyte hormone adiponectin. Proc. Natl Acad. Sci. USA doi:10.1073/ pnas.1415219111 